\section{AB0601 COMPARISON OF THE EFFECT OF DIFFERENT PHYSICAL THERAPY PROGRAM IN THE REHABILITATION OF KNEE OSTEOARTHRITIS PATIENTS}

A. Vizdoaga ${ }^{1}$, V. Salaru ${ }^{2}$, M. Cebanu ${ }^{3}$, V. Sadovici-Bobeica ${ }^{4}$, N. Loghin-Oprea ${ }^{4}$, L. Mazur-Nicorici ${ }^{4}$, T. Rotaru ${ }^{4}$, M. Garabajiu' ${ }^{2}$, M. Mazur ${ }^{4} .{ }^{1}$ Nicolae Testemitanu State University of Medicine and Pharmacy, University Medical Rehabilitation Center, Chisinau, Moldova, Republic of: ${ }^{2}$ Nicolae Testemitanu State University of Medicine and Pharmacy, Family Medicine, Chisinau, Moldova, Republic of; ${ }^{3}$ Nicolae Testemitanu State University of Medicine and Pharmacy, Emergency medicine, Chisinau, Moldova, Republic of; ${ }^{4}$ Nicolae Testemitanu State University of Medicine and Pharmacy, Internal Medicine, Chisinau, Moldova, Republic of

Background: Knee osteoarthritis $(\mathrm{OA})$ is an increasingly common and disabling problem in worldwide. Exercises are considered the cornerstone of non-surgical management of knee $\mathrm{OA}$ and is recommended in all current clinical guidelines. The diversity of exercise programs determines the need to compare their efficiency, in order to recommend the best option for the patient.

Objectives: The aim of the present study was to compare the effect on knee functionality of conventional exercises program and combinate with manual therapy in patients with knee osteoarthritis.

Methods: 164 patients that fulfilled the ACR classification criteria for knee osteoarthritis (127 females and 37 males) ranging in age from 42 to 84 years (mean 62,2 SD 8,76), participated in a 10 days two-arm randomized trial. One group (Gr.1) received an exercises program, the other group additionally manual therapy methods (Gr.2). Pain and function were measured with a Visual Analogue Scale (VAS, $\mathrm{mm}$ ) and Knee Injury and Osteoarthritis Outcomes Score (KOOS) with 5 domains (Pain, Symptoms, Activity in Daily Living (ADL), Sport and recreation (Sport/rec.) and Quality of life (QoL)).

Results: In the Gr.1 were 82 patients mean age $61,8 \pm 9,2$ years and in Gr.2 - 82 patients $62,7 \pm 8,3$ years $(p>0.05)$. The initial level of pain $(63,4 \pm 14,2$ vs $60,2 \pm 18,8 \mathrm{~mm}$ ) and knee functionality according to KOOS (Tab1.) were similar in both groups, $p>0.05$. After 10 days rehabilitation program, VAS in Gr.1 was $39,6 \pm 14,8 \mathrm{~mm}$, in Gr.2- $28,5 \pm 11,6 \mathrm{~mm},(p<0.01)$. The KOOS results at the end of the rehabilitation program were with improvement, statistically significative in the Gr.2 ( $p<0,05)$.

Table 1. The KOOS results at baseline and after 10 days of rehabilitation program.

\begin{tabular}{lcccc}
\hline KOOS scales & \multicolumn{2}{c}{ Group 1 } & \multicolumn{2}{c}{ Group 2 } \\
\cline { 2 - 5 } (M \pm SD), \% & Baseline & After 10 days & Baseline & \multirow{2}{*}{ After 10 days } \\
\hline Pain & $55,5 \pm 13,8$ & $63,4 \pm 13,6$ & $53,0 \pm 22,3$ & $64,3 \pm 19,1$ \\
Symptoms & $59,0 \pm 18,7$ & $65,6 \pm 18,1$ & $56,6 \pm 19,0$ & $69,0 \pm 15,9$ \\
ADL & $46,3 \pm 15,5$ & $54,5 \pm 16,0$ & $43,0 \pm 19,9$ & $55,1 \pm 18,2$ \\
Sport/rec & $15,6 \pm 17,3$ & $23,1 \pm 19,6$ & $16,0 \pm 24,3$ & $30,5 \pm 23,6$ \\
QoL & $29,3 \pm 22,2$ & $36,5 \pm 22,9$ & $34,8 \pm 23,9$ & $50,6 \pm 18,9$ \\
\hline
\end{tabular}

Conclusion: The reduction of pain and recovery of function were found in both groups, with better results in patients who received manual therapy and exercises program. This study supports the routine manual therapy in rehabilitation program in patients with knee osteoarthritis.

Disclosure of Interests: None declared.

DOI: 10.1136/annrheumdis-2021-eular.3570

\section{AB0602 $1 M P A C T$ OF SOCIOECONOMIC FACTORS ON PAIN AND FUNCTION IN KNEE OSTEOARTHRITIS: A SYSTEMATIC LITERATURE REVIEW}

T. W. Swinnen ${ }^{1}$, M. Willems ${ }^{2}$, I. Jonkers ${ }^{2}$, F. P. Luyten ${ }^{3}$, J. Vanrenterghem ${ }^{1}$, S. Verschueren ${ }^{1}{ }^{1} \mathrm{KU}$ Leuven, Research Group for Musculoskeletal Rehabilitation, Department of Rehabilitation Sciences, Leuven, Belgium; ${ }^{2} \mathrm{KU}$ Leuven, Human Movement Biomechanics Research Group, Department of Movement Sciences, Leuven, Belgium; ${ }^{3} \mathrm{KU}$ Leuven, Skeletal Biology and Engineering Research Center, Leuven, Belgium

Background: The personal and societal burden of knee osteoarthritis (KOA) urges the research community to identify factors that predict its onset and progression. A mechanistic understanding of disease is currently lacking but needed to develop targeted interventions. Traditionally, risk factors for KOA are termed 'local' to the joint or 'systemic' referring to whole-body systems. There are however clear indications in the scientific literature that contextual factors such as socioeconomic position merit further scientific scrutiny, in order to justify a more biopsychosocial view on risk factors in KOA.
Objectives: The aims of this systematic literature review were to assess the inclusion of socioeconomic factors in KOA research and to identify the impact of socioeconomic factors on pain and function in KOA.

Methods: Major bibliographic databases, namely Medline, Embase, CINAHL, Web of Science and Cochrane, were independently screened by two reviewers (plus one to resolve conflicts) to identify research articles dealing with socioeconomic factors in the KOA population without arthroplasty. Included studies had to quantify the relationship between socioeconomic factors and pain or function. Main exclusion criteria were: a qualitative design, subject age below 16 years and articles not written in English or Dutch. Methodological quality was assessed via the Cochrane risk of bias tools for randomized (ROB-II) and non-randomized intervention studies (ROBIN-I) and the Newcastle-Ottawa Scale for assessing the quality of non-randomised studies. Due to heterogeneity of studies with respect to outcomes assessed and analyses performed, no meta-analysis was performed.

Results: Following de-duplication, 7639 articles were available for screening (120 conflicts resolved without a third reader). In 4112 articles, the KOA population was confirmed. 1906 (25\%) were excluded because of knee arthroplasty and $1621(21 \%)$ because of other issues related to the population definition. Socioeconomic factors could not be identified in $4058(53 \%)$ papers and were adjusted for in $211(3 \%)$ articles. In the remaining papers covering pain $(n=110)$ and/o function $(n=81)$, education $(62 \%)$ and race $(37 \%)$ were most frequently assessed as socioeconomic factors. A huge variety of mainly dichotomous or ordinal socioeconomic outcomes was found without further methodological justification nor sensitivity analysis to unravel the impact of selected categories. Although the Western Ontario and McMaster Universities Osteoarthritis Index (WOMAC) was the most popular instrument to assess pain and function, data pooling was not possible as socioeconomic factors estimates were part of multilevel models in most studies. Overall results showed that lower education and African American race were consistent predictors of pain and poor function, but those effects diminished or disappeared when psychological aspects (e.g. discrimination) or poverty estimates were taken into account. When function was assessed using self-reported outcomes, the impact of socioeconomic factors was more clear versus performance-based instruments. Quality of research was low to moderate and the moderating or mediating impact of socioeconomic factors on intervention effects in KOA is understudied.

Conclusion: Research on contextual socioeconomic factors in KOA is insufficiently addressed and their assessment is highly variable methodologically Following this systematic literature review, we can highlight the importance of implementing a standardised and feasible set of socioeconomic outcomes in KOA trials ${ }^{1}$, as well as the importance of public availability of research databases including these factors. Future research should prioritise the underlying mechanisms in the effect of especially education and race on pain and function and assess its impact on intervention effects to fuel novel (non-)pharmacological approaches in KOA.

\section{REFERENCES:}

[1] Smith TO et al. The OMERACT-OARSI Core Domain Set for Measurement in Clinical Trials of Hip and/or Knee Osteoarthritis J Rheumatol 2019. 46:981-9. Disclosure of Interests: None declared. DOI: 10.1136/annrheumdis-2021-eular.3634

\section{$\mathrm{AB} 0603$ THE NEW POSSIBILITIES OF USING CHONDROITIN SULFATE IN PATIENTS WITH KNEE OSTEOARTHRITIS}

E. Sharapova ${ }^{1}$, N. Kashevarova ${ }^{1}$, E. Taskina ${ }^{1}$, D. Kusevich ${ }^{1}$, L. Alekseeva ${ }^{1,2}$ A. Lila ${ }^{2,3}{ }^{1}$ V. A. Nasonova Research Institute of Rheumatology, Laboratory of Osteoarthritis, Moscow, Russian Federation; ${ }^{2}$ Russian Medical Academy of Continuous Professional Education, Rheumatology, Moscow, Russian Federation; ${ }^{3}$ V. A. Nasonova Research Institute of Rheumatology, director, Moscow, Russian Federation

Background: Administration of Chondroitin Sulfate for treatment of osteoarthritis included treatment types include systemic and parenteral therapies. The benefits of local therapies between difference route is ambivalent.

Objectives: To evaluate effectiveness and safety of Chondroitin Sulfate (CS) by intramuscular and concomitant (intramuscular and intraarticular) route of administration in patients with knee osteoarthritis $(\mathrm{OA})$.

Methods: In total 150 patients with knee OA according by ACR criteria and with radiological stages 2 and 3 according to Kellgren-Lawrence were enrolled. Patients were divided by 2 groups: 75 patients $(R)$ received course of 25 intramuscular injection every other day: at the first 3 injection's in dose $100 \mathrm{mg}$, and in continue in dose $200 \mathrm{mg}, 75$ patients in 2 nd group $(\mathrm{N})$ received 5 intra-articular injections in target knee in dose $200 \mathrm{mg}$ once per 3 days, in continue 16 intramuscular injections every other day in dose $200 \mathrm{mg}$. All patients received NSAID - meloxicam in stable dose $15 \mathrm{mg}$ per day. All patients were checked by WOMAC, pain by visual analog scale (VAS), McGill Pain Questionnaire (MPQ) 